\title{
Validated predictive computational methods for surface charge in heterogeneous functional materials: HeteroFoaM ${ }^{\mathrm{Tm}}$
}

Kenneth L Reifsnider*, Dan G Cacuci, Jeffrey Baker, Jon Michael Adkins and Fazle Rabbi

\begin{abstract}
Background: Essentially all heterogeneous materials are dielectric, i.e., they are imperfect conductors that generally display internal charge displacements that create dissipation and local charge accumulation at interfaces. Over the last few years, the authors have focused on the development of an understanding of such behaviour in heterogeneous functional materials for energy conversion and storage, called HeteroFoaM (www.HeteroFoaM.com). Using paradigm problems, this work will indicate major directions for developing generally applicable methods for the multiphysics, multi-scale design of heterogeneous functional materials.
\end{abstract}

Methods: The present paper outlines the foundation for developing validated predictive computational methods that can be used in the design of multi-phase heterogeneous functional materials, or HeteroFoaM, as a genre of materials. Such methods will be capable of designing not only the constituent materials and their interactions, but also the morphology of the shape, size, surfaces and interfaces that define the heterogeneity and the resulting functional response of the material system.

Results: Relationships to applications which drive this development are identified. A paradigm problem based on dielectric response is formulated and discussed in context.

Conclusions: We report an approach that defines a methodology for designing not only the constituent material properties and their interactions in a heterogeneous dielectric material system, but also the morphology of the shape, size, surface, and interfaces that defines the heterogeneity and the resulting functional response of that system.

Keywords: Heterogeneous materials; Dielectric; Surface charge; Predictive modelling; Computational methods

\section{Background}

\section{Heterogeneous materials}

Essentially all heterogeneous materials are dielectric, i.e., they are imperfect conductors that generally display internal charge displacements that create dissipation and local charge accumulation at interfaces. Over the last few years, the authors have focused on the development of an understanding of such behaviour in heterogeneous functional materials for energy conversion and storage, called HeteroFoaM (www.HeteroFoaM.com). Using paradigm problems, this work will indicate major directions for developing generally applicable methods for the multiphysics, multi-scale design of heterogeneous functional materials.

\footnotetext{
* Correspondence: Reifsnider@sc.edu

University of South Carolina, Columbia, SC 29209, USA
}

Heterogeneous functional materials are widely used in our society as the materials systems that make batteries, fuel cells, separation membranes, and many electrochemical devices possible. They typically function by operating on the fields applied to them to convert, transport, or store energy from applied chemical, electrical, mechanical, or thermal conditions. Figure 1 shows several examples of the applications that drive the development of such material systems. Figure 1(b) and (d) show particulate systems as used in fuel cells and high temperature membrane systems. Figure 1(b) shows an example of an anode of a solid oxide fuel cell (SOFC) which consists (principally) of particles that are electronic conductors, particles that are ionic conductors, and a connected void phase that transports fuel to the SOFC for conversion into electricity. This conversion requires that fuel gas be transported through 

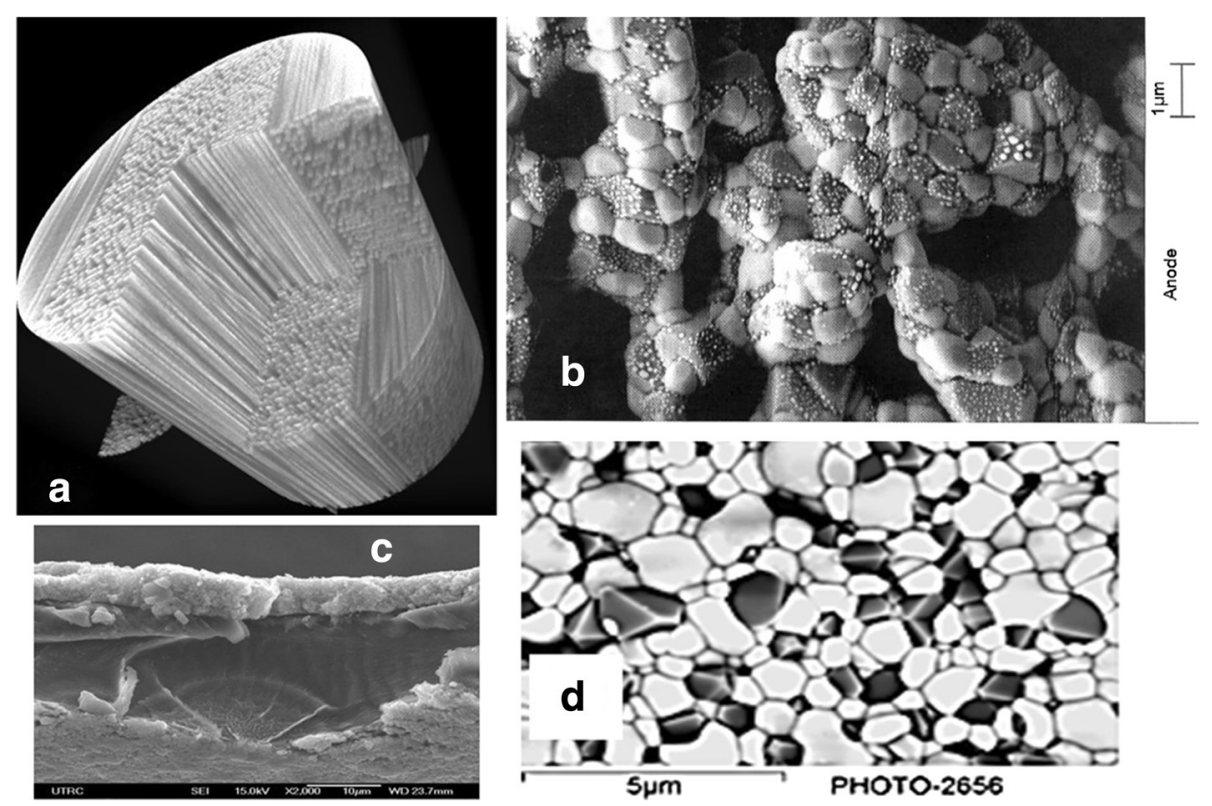

Figure 1 Heterogeneous functional materials used in aerospace structures (a), solid oxide fuel cells (b), polymer membrane fuel cells (c) and ceramic separation membranes for oxygen transport (d).

the pore phase, adsorbed and oxidized to generate electrons which are conducted away through the external circuit (to drive an electric load), while oxygen ions are transported by the second solid phase (from the cathode side of the SOFC) to combine with the hydrogen (protons) to form $\mathrm{H}_{2} \mathrm{O}$, the product gas, which is transported away. But the electrochemical reaction just described requires a "triple point boundary" where the ionic and electronic conductors are exposed to the hydrogen containing fuel gas to enable the chemical reaction that drives the energy conversion process. Triple point boundaries in Figure 1 are defined by the interfaces of the light and dark particles that are exposed to the void phase.

Although similar discussions could be advanced for the other microstructures shown in Figure 1, the SOFC example exemplifies the problem addressed in the present paper. The computational methods that we envisage developing for the design of HeteroFoaM systems must provide a foundation for the design of all of the local details of microstructure as well as support and represent the controlling physics that drives the functionality of the materials system. Several computational methods for these general problems have already been developed and will be discussed (Liu 2012; Liu 2011a; Liu \& Reifsnider 2013; Reifsnider et al. 2013). An extensive experimental program has been conducted to validate our understanding at the fundamental level (Baker et al. 2014). However, the charge that accumulates at the material boundaries in such heterogeneous material mixtures is not yet fully described and understood, and provides the focus of the present paper.

\section{Methods}

We consider a simple two-phase material with morphology as shown in Figure 2 as a foundation for describing our approach. We require that we be able to determine the variation of sensible potential at all points and boundaries caused by the application of a vector electric field, which may be static or harmonic. Figure 2 shows the direction of the vector electric field. The paradigm problem depicted in Figure 2(a) and (b) is one dimensional, i.e., the material in our domain (and all applied conditions) are constant to infinity in the vertical direction; while Figure 2(c) depicts a two dimensional problem with boundary conditions to be discussed in the sequel.

For the purposes of this paper, we consider only the conservation of charge: the charge displacement due to dielectric polarization, $D$, generates the flux, $\rho$, so that $\rho=\nabla \cdot D$, where $\mathrm{D}$ is related to the applied field by the permittivity of the $\mathrm{k}^{\text {th }}$ phase, $\varepsilon_{\mathrm{k}}$ according to the relationship $D=\epsilon_{o} \cdot \epsilon_{k} \cdot E$ (where $\epsilon_{o}$ is the permittivity of vacuum), and the charge flux caused by conduction, $\mathrm{J}$, is related to the applied field, $\mathrm{E}$, by $J=\sigma \cdot E$ where $\sigma$ is the conductivity. Neglecting source terms, we model the conservation of charge in the form

$$
\nabla \cdot J+\frac{d \rho}{d t}=0, \quad \rightarrow \quad \nabla \cdot\left(J+\frac{\partial D}{\partial t}\right)=0
$$




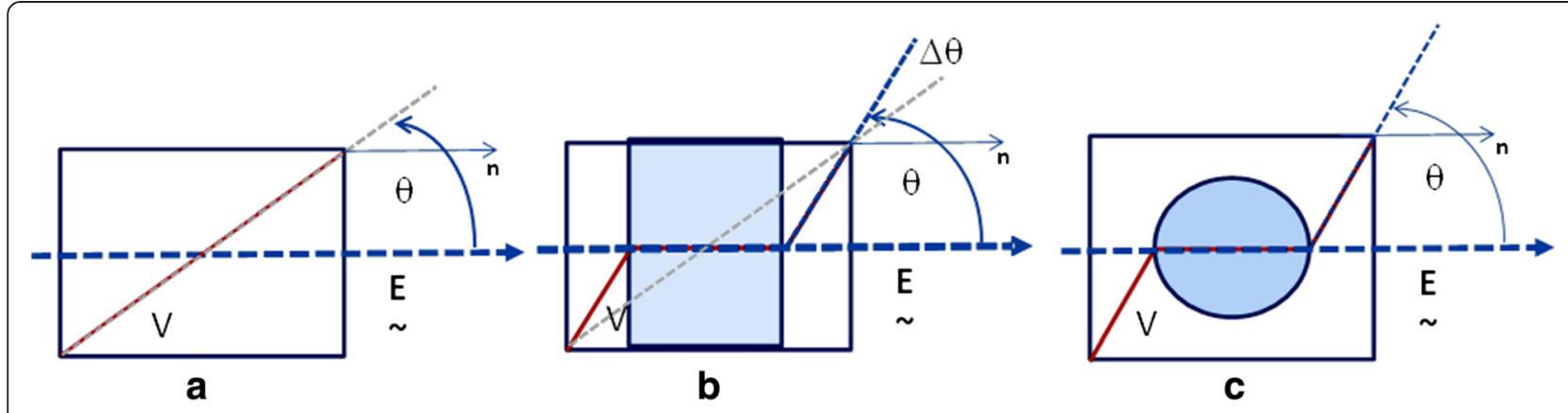

Figure 2 Representative volume elements for the design of a dielectric heterogeneous material with two phases: (a) plate of infinite extent in the vertical direction, vector electric field applied across boundaries in the horizontal direction to uniform interior material; (b) same as case (a) except that a second phase material is added in the interior (shaded region); (c) 2-D domain with prescribed boundary conditions on the top and bottom (e.g., symmetrical/repeating, insulating, etc.) and a uniform applied field in the horizontal direction.

Recognizing that the field, E, is the gradient of the sensible local potential, $V$ it follows that

$$
\nabla \cdot(\sigma \nabla \mathrm{V})+\frac{\mathrm{d}}{\mathrm{dt}} \nabla\left(\epsilon \epsilon_{o} \nabla V\right)=0
$$

If the applied field is harmonic, proportional to $e^{-j \omega t}$, then Eq. (2) takes on a form that is typically solved by codes such as COMSOL (Ref ?), namely

$$
-\nabla \cdot d\left[\left(\sigma+j \omega \varepsilon \varepsilon_{0}\right) \nabla V-\left(J^{c}+j \omega P\right)\right]=d Q_{j}
$$

where $\sigma$ and $\varepsilon$ are the conductivity and permittivity, respectively, of a given phase, $\omega$ is the frequency of oscillation of the electric field, $\nabla$ is the gradient operator, $j \equiv \sqrt{-1}, P$ is the polarization, while $J^{c}$ and $Q_{j}$ denote source terms. In a series of previous publications, we have applied equation (3) to some of the complex microstructures illustrated in Figure 1 at the conformal level, and have included electrochemical effects on the resulting flux terms shown in that equation. However, in this publication, we are only concerned with constructing a predictive method for time dependent electric charge distribution (including surface charge) in heterogeneous materials at the fundamental level, and the validation of such a method (Liu 2012; Liu 2011a; Liu \& Reifsnider 2013; Reifsnider et al. 2013; Baker et al. 2014; Liu 2011b; Raihan et al. 2014). Therefore, without loss of generality for the present discussion, we neglect all source terms and concern ourselves with the following simplified version of Eq. (1):

$$
-\nabla \cdot d\left[\left(\sigma+j \omega \varepsilon_{0}\right) \nabla V\right]=0
$$

where $d$ denotes the thickness of our domain perpendicular to the diagram. There are many forms of the material constants in Eq. (2), but generally they may have both real (in phase) and imaginary (out of phase) components. If we consider a transcendental form for $\varphi$ and take only the real part of the material property coefficient in Eqs. (2) and (4), one could consider the form

$$
\nabla \cdot d[(\sigma-\omega \varepsilon \sin (\omega \mathrm{t}) \nabla V]=0,
$$

or, for our physical discussion, taking $(d=1)$ and the modulus of the transcendental term to be of order one, we simply discuss the design equation

$$
\nabla \cdot[(\sigma-\omega \varepsilon) \nabla V]=0 .
$$

\section{Results and discussion: forward problem}

These forms help us to define the local physics that is to be specified in our design of the material system, and help us to properly set a design analysis. Although we are considering only a balance of charge in the present case, the analysis can be extended to other conservation equations (e.g., for mass, momentum, and energy) to support a multiphysics design with a more general discussion. However, the present discussion will adequately define the methodology for more general applications.

At the most general level, examination of the physical domain in Figure 2 together with Eqs. (1) - (6) indicate that for an applied vector electric field, the slope of the potential in that direction multiplied by a material constant which controls the charge displacement in that material is constant, as we know and expect from electrostatics. So in Figure 2(a) for a uniform material the sensible voltage, $V$, is a straight line across the material. If the material is an ideal conductor, the slope of that line is simply the conductivity of the material, $\sigma$, for the static case. However, if the material has some permittivity, the slope of $V$ may be greater or smaller at various 
frequencies of the input field; at very high input frequencies, the system acts like a parallel plate capacitor, as it must, and the slope of the potential $(V)$ across the plates approaches zero.

Before leaving this simple case, we note that our design is not only concerned with the variation of the potential from point to point. More generally, we are asked to design the microstructure to control the flux and the polarization, i.e., to control the charge distribution in the heterogeneous system, for minimizing, e.g., the dissipation and losses in electrical devices or for enhancing the storage of charge in energy storage devices like capacitors or batteries. To accomplish such goals, we must design the surface charge that defines the polarizations when the electric field is applied to a multi-phase material. In Figure 2(b), we introduce an infinite plate in the gap between our applied voltages on the left and right boundaries of our domain in Figure 2 (a). In Figure 2(b), the second phase has a higher conductivity (and/or permittivity) than the "matrix" phase around it, so that the slope of the potential across that region is smaller than in the surrounding material. Such a change also affects the surface charge in the system. At the interface between two phases, the voltage must remain continuous across the boundary, i.e., $V^{i}=V^{o}$ and

$$
\varepsilon^{o} \frac{d V^{o}}{d n}-\varepsilon^{i} \frac{d V^{i}}{d n}=\sigma_{f}
$$

where $\sigma_{f}$ denotes the free charge across an interface, $d / d n$ denotes the derivative along the normal to the interface surface, while the superscripts " $o$ " and " $i$ " denote the "outside" and "inside" material regions, respectively. Since we are not concerned with free charge in this paper, we can set $\sigma_{f}=0$ in Eq. (7). Using Gauss's law to calculate the total interface charge density across a heterogeneous material interface yields

$$
-\varepsilon^{o} \frac{d V^{o}}{d n}+\varepsilon^{i} \frac{d V^{i}}{d n}=\sigma_{t}
$$

As we can see from Figure 2, and as is well known, introducing a dielectric (or conductor) in the region between the "parallel plates" of Figure 2(a) creates a larger change in slope at the boundary and also a surface charge at the interior interface, resulting in an increase in charge storage and resulting capacitance of the domain (Liu 2011b). But the extrapolation of this simple reasoning to the design of more general geometries and, eventually, more complex microstructures is not a straightforward extension of these simple rationales.

The next simplest design situation is a two dimensional heterogeneous domain in which the included phase is a simple cylinder, as shown in Figure 2(c). In this case, the domain is finite in the plane of observation, a square in cross section for example, and all variables are constant along the axis in and out of the plane of the figure. The equations have the same formal appearance but the material properties are discontinuous across the curved boundary between the included phase (the "inside phase" in our publications), and the matrix phase around it (the "outside phase"). Gauss' law indicates that the surface charge on the included surface of the inclusion is proportional to the local change in slope of the potential in the direction of the surface normal, i.e., proportional to the change in local field in the material in that direction. But now the field direction is not constant w.r.t. the surface normal direction, and therefore, the surface charge varies around the circumference of the cylindrical inclusion. In earlier calculations we have determined that distribution, as shown for the example calculations in Figure 3 (Liu 2012; Liu 2011a; Liu \& Reifsnider 2013; Reifsnider et al. 2013). Moreover, we have confirmed that the predicted potential distributions for circular and elliptical second phase inclusions (for various orientations to the vector electric field direction) are correct from direct measurements with an atomic force microscope (Baker et al. 2014). Our data confirm that for highly conductive inclusion in a poorly conductive, high permittivity matrix (epoxy in this case), the time dependent response shown in Figure 4 is observed.

If we were to use this analysis approach for design, we could, in principle, "run the forward problem" many times, for many candidate morphologies, and invoke a variety of optimization methods to determine "what the picture should look like". Although for simple singlephysics problems such an approach might be feasible; such an approach for a multi-physics analysis would be akin to the many body problem (which often involves additional nonlinear response associated with coupling, etc.), which would quickly become computationally intractable and unpredictable.

\section{Results and discussion: design for response}

The "direct" or "forward" problem" solves the "parameterto-output" mapping that describes the "cause-to-effect" relationship in the respective physical process. Taking Eq. (3), for example, the "forward (or direct) problem" consists in solving it subject to appropriate boundary conditions to obtain the solution $V(\mathbf{x})$, which is in turn used to compute the model responses $\mathbf{r}[V(\mathbf{x}) ; \boldsymbol{\alpha}(\mathbf{x})]$, where $\boldsymbol{\alpha}(\mathbf{x}) \equiv\left[d(\mathbf{x}), \sigma(\mathbf{x}), \omega, \varepsilon(\mathbf{x}), J^{c}(\mathbf{x}), P(\mathbf{x}), Q_{j}(\mathbf{x})\right]$ denotes the vector of spatially-( $\mathbf{x}$-)dependent model parameters. The necessary and sufficient conditions for the direct problem to be well-posed were formulated by Hadamard (1865-1963), and can be stated as follows: (i) For each source, $Q_{j}$, there exists a solution $V(\mathbf{x})$; (ii) The solution $V(\mathbf{x})$ is unique; (iii) The dependence of $V(\mathbf{x})$ upon "the 


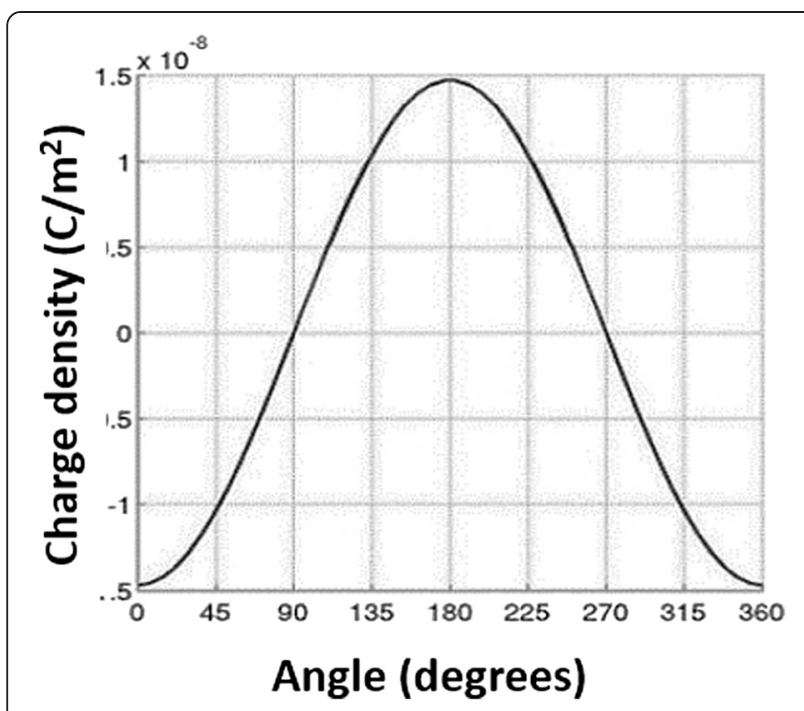

Figure 3 Calculated charge distribution around the circum-ference of a circular inclusion as a function of angle to the direction of the applied vector electric field.

data" $\boldsymbol{\alpha}(\mathbf{x})$ and boundary conditions is continuous. A problem that is not well-posed is called ill-posed. In general, two problems are called inverses of one another if the formulation of each involves all or part of the solution of the other. Several inverse problems can be formulated, as follows: (a) The classical "inverse source identification problem": given the responses $\mathbf{r}$, the known boundary conditions, and the parameters $\boldsymbol{\alpha}(\mathbf{x})$, determine the sources $Q_{j}$; (b) "Parameter identification problem": given the responses $\mathbf{r}$ and the sources $Q_{j}$, determine the parameters $\boldsymbol{\alpha}(\mathbf{x})$; (c) When the domain contains inhomogeneous materials, and the responses $\mathbf{r}$ are given, identify internal boundaries between the

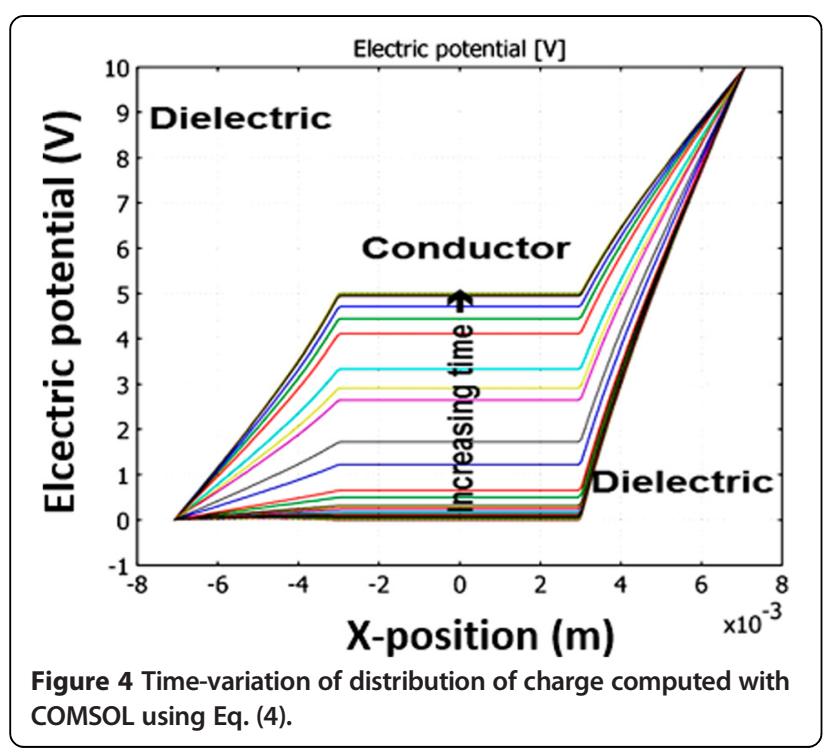

inhomogeneous materials, identify the description of the system's structure ("structural identification"), etc.

The existence of a solution for an inverse problem is, in most cases, secured by defining the data space to be the set of solutions to the direct problem. This approach may fail if the data is incomplete, perturbed or noisy. Problems involving differential operators, for example, are notoriously ill-posed, because the differentiation operator is not continuous with respect to any physically meaningful observation topology. If the uniqueness of a solution cannot de secured from the given data, additional data and/or a priori knowledge about the solution need to be used to further restrict the set of admissible solutions. Of the three Hadamard requirements, stability is the most difficult to ensure and verify. If an inverse problem fails to be stable, then small round-off errors or noise in the data will amplify to a degree that renders a computed solution useless.

Designing "HeteroFoaM" materials is fundamentally an inverse problem. The difficulties that must be overcome when developing validated predictive computational methods for designing multi-phase heterogeneous functional materials (HeteroFoaM) can be illustrated by considering the "inverse source identification problem" for a one-dimensional domain extending from $x=0$ to $x=d$ (considered to be infinite in the $y$ - and $z$-directions), and having perfectly known constant material properties, in which the potential, $V(x)$, is driven by a spatially varying source, $Q(x) \equiv d Q_{j}$. For such an idealized material, Eq. (3) takes on the simple form

$$
\begin{gathered}
-A d^{2} V(x) / d x^{2}=Q(x), 0<x<d, \\
\text { with } A \equiv d\left(\sigma+j \omega \varepsilon \varepsilon_{0}\right), Q(x) \equiv d Q_{j} .
\end{gathered}
$$

The "inverse source determination problem" is to determine the source $Q(x)$ from measurements of the potential, $V(x)$. A measurement would be recorded as a "detector" or "instrument response" that can be represented in the form

$$
M \equiv \int_{0}^{d} V(x) R_{d}(x) d x
$$

where $R_{d}(x)$ represents the detector's response function. For a known (measured) response value $M$, it is evident that Eq. (10) represents a Fredholm equation of the first kind for the determination of the spatially-dependent voltage, $V(x)$, which cannot be solved as it stands to produce a unique solution $V(x)$ ! Moreover, Fredholm equations of the first kind are notoriously ill-posed, since the integration over the 
kernel [in this case, $R_{d}(x)$ ] of the Fredholm equation has a "smoothing" effect on the high-frequency components, and may include cusps, and edges in $V(x)$. This effect stems from the well-known RiemannLebesgue lemma, which for the purposes of this work can be written in the form

$$
\lim _{\omega \rightarrow \infty} \int_{a}^{b} f(x) e^{j \omega x} d x \rightarrow 0, \text { with } f(x)=\text { piecewise continuous. }
$$

It can therefore be expected that the (inverse) determination of $V(x)$ using the Fredholm Eq. (10) will amplify high frequency components (such as those stemming from measurement errors) in the measured "detector response" $M$.

In practice, the Fredholm equation (10) must be discretized in order to determine $V(x)$. There are two main classes of methods for discretizing integral equations, namely quadrature methods and Galerkin (which include collocation, spectral, and pseudo-spectral) methods. Consider, for simplicity, that

$$
R_{d}(x)=C_{d} \delta\left(x-x_{n}\right)
$$

where $C_{d}$ is an appropriate "measurement conversion function", so that the detector provides, at any spatial location $x_{n}$, a measurement of the form

$$
M_{n} \equiv C_{d} V\left(x_{n}\right), n=0,1, \ldots, N .
$$

On the other hand, in order for Eq. (9) to make physical sense, it is clear that the function $V(x)$ must be square integrable, piecewise continuous, and of bounded variation (having at most a finite set of discontinuities of finite magnitudes within the slab). Therefore, the function $V(x)$ must admit a spectral (e.g., Fourier) representation, and the choice of basis-functions can be conveniently adapted to boundary conditions and possible periodicities and/or symmetries inherent in the problem under consideration. For our illustrative inverse problem, we expect to be able to measure $V(x)$ at least at the left and right boundaries of the slab, i.e., at $x=0$, and at $x=d$, respectively, obtaining two values, which will be conveniently denoted as $M_{0} \equiv C_{d} V(0)$ and $M_{N}=C_{d} V\left(x_{N}\right), x_{N} \equiv d$, respectively. From the point of view of the forward problem, these measurements would mathematically provide (two) Dirichlet boundary conditions for $V(x)$, namely

$$
V(0)=C_{d} / M_{0}, V\left(x_{N}\right)=C_{d} / M_{N},
$$

to complement the differential equation (9), thus rendering the forward problem [namely, to determine the function $V(x)$ when the source $Q(x)$ is known] to be perfectly well-posed in the sense of Hadamard. Actually, the unique and exact solution, $V^{\text {exact }}(x)$, of the forward problem consisting of Eqs. (9) and (14), is

$$
V^{\text {exact }}(x)=\sum_{n=1}^{\infty} c_{n}^{\text {exact }} \sin \frac{n \pi x}{d}
$$

with the coefficients $c_{n}^{\text {exact }}$ given by the expression

$$
\begin{aligned}
& c_{n}^{\text {exact }} \equiv \frac{n \pi / d\left[(-1)^{n+1} V\left(x_{N}\right)+V(0)\right]+q_{n} / A}{(n \pi / d)^{2}}, \\
& q_{n} \equiv \int_{0}^{d} Q(x) \frac{n \pi x}{d} d x
\end{aligned}
$$

Returning now to the inverse problem at hand, it becomes clear that the spectral representation shown in Eq. (15) underscores that fact the determination of $V^{e x a c t}$ $(x)$ would require infinitely many measurements, $M_{n}$ in order to determine all of the coefficients $c_{n}^{\text {exact }}$. But it is practically impossible to perform infinitely many measurements. In practice, therefore, the determination of the first $J$ coefficients $\left(c_{1}, \ldots, c_{J}\right)$ necessitates $J$ measurements of $V(x)$ at locations $\left(x_{1}, \ldots, x_{J}\right)$, in order to construct the following system of equations (for determining the coefficients $c_{1}, \ldots, c_{J}$ ):

$$
\begin{aligned}
& \mathbf{S C}=\mathbf{M}, \quad \mathbf{S} \equiv\left(\begin{array}{ccc}
\sin \frac{\pi x_{1}}{d} & \ldots & \sin \frac{J \pi x_{1}}{d} \\
\vdots & \ddots & \vdots \\
\sin \frac{\pi x_{J}}{d} & \cdots & \sin \frac{J_{\pi x_{J}}}{d}
\end{array}\right), \\
& \mathbf{C} \equiv\left(\begin{array}{c}
c_{1} \\
\vdots \\
c_{J}
\end{array}\right) ; \quad \mathbf{M} \equiv\left(\begin{array}{c}
M_{1} \\
\vdots \\
M_{J}
\end{array}\right) .
\end{aligned}
$$

Solving the above system yields the coefficients $\left(c_{1}, \ldots, c_{J}\right)$ as the solution of the equation

$$
\mathbf{C}=\mathbf{S}^{-1} \mathbf{R}
$$

It is clear from the foregoing considerations that the $\mathrm{co}^{-}$ efficients $c_{n}$ cannot possibly be determined perfectly, for at least the following three reasons: (i) it is impossible to perform infinitely many measurements; (ii) the measurements $M_{j}$ cannot be performed perfectly, so they will be afflicted by measurement errors; and (iii) inverting the matrix $\mathbf{S}$ will introduce additional numerical errors. Therefore, the reconstructed coefficients $c_{n}$ will be affected by errors, which can be considered to be additive, of the form

$$
c_{n}=c_{n}^{\text {exact }}+\varepsilon_{n} .
$$

where $c_{n}^{\text {exact }}$ denotes the exact, but unknown $n^{\text {th }}$-coefficient, while $\varepsilon_{n}$ denotes the corresponding error. Hence, 
the reconstructed potential, denoted here as $V^{\text {rec }}(x)$, will have the form

$$
V^{r e c}(x)=\sum_{n=1}^{J} c_{n}^{\text {exact }} \sin \frac{n \pi x}{d}+\sum_{n=1}^{J} \varepsilon_{n} \sin \frac{n \pi x}{d} .
$$

The above representation of the potential clearly indicates that its reconstruction from measurements introduces errors over the entire spatial-frequency spectrum. It is especially important to note that the highest-frequency spatial errors cannot be controlled from the "measurement side" since they arise precisely because of the truncation to finitely many terms, which actually stems from the inability to perform infinitely many measurements.

The effects of the errors displayed in Eq. (19) on attempting to determine the source $Q(x)$ from Eq. (9) can now be displayed explicitly, as follows. If the exact expression, $V^{\text {exact }}(x)$, given in Eq. (15) were available, if the model represented by Eq. (9) were perfect, and if the boundary values given in Eq. (14) were perfectly well known, then the exact expression for the source, $Q^{\text {exact }}(x)$, could be obtained by replacing Eq. (15) into Eq. (9). The expression thus obtained for $Q^{\text {exact }}(x)$ would be

$$
Q^{\text {exact }}(x)=A \sum_{n=1}^{\infty} c_{n}^{\text {exact }}\left(\frac{n \pi}{d}\right)^{2} \sin \frac{n \pi x}{d},
$$

where the exact coefficients $c_{n}^{\text {exact }}$ would decay sufficiently fast, as functions of $n$, to ensure the convergence of the infinite series on the right side of Eq. (20). This property can be readily verified by using Eq. (16) to compute the exact coefficients, $c_{n}^{\text {exact }}$, that would result from various particular forms of the source $Q^{\text {exact }}(x)$.

However, the exact potential $V^{\text {exact }}(x)$, is unavailable! Only the reconstructed potential, $V^{r e c}(x)$, given in Eq. (20) is available. Replacing this expression into Eq. (9) yields

$$
\begin{aligned}
A \sum_{n=1}^{J} c_{n}^{\text {exact }}\left(\frac{n \pi}{d}\right)^{2} \sin \frac{n \pi x}{d} & +A \sum_{n=1}^{J} \varepsilon_{n}\left(\frac{n \pi}{d}\right)^{2} \sin \frac{j \pi x}{d} \\
& =Q^{\text {exact }}(x)+Q^{\text {error }}(x, J),
\end{aligned}
$$

where

$$
Q^{\text {error }}(x, J) \equiv A \sum_{n=1}^{J} \varepsilon_{n}\left(\frac{n \pi}{d}\right)^{2} \sin \frac{n \pi x}{d}-A \sum_{n=J+1}^{\infty} c_{n}^{e x}\left(\frac{n \pi}{d}\right)^{2} \sin \frac{n \pi x}{d} .
$$

Even though the exact values of the coefficients $\varepsilon_{j}$ are unknown, they are nevertheless some numerical constants, and the crucial fact is that they do not depend on $n$. It therefore follows that, in the limit of large $J$, the second sum in Eq. (23) will vanish, but the first sum will diverge to infinity, so that

$$
\lim _{J \rightarrow \infty} Q^{\text {error }}(x, J) \rightarrow \infty .
$$

The above behavior of $Q^{\text {error }}(x, J)$ clearly highlights the destructive effect of high frequency errors when attempting to determine the source from flux measurements by using the forward Eq. (3): high-frequency error components arising from the reconstruction of the flux from measurements cause a large deviation between the true source and the source that would be reconstructed from flux measurements. Furthermore, this discrepancy between the true and the reconstructed source is the larger the higher the frequency of the error component in the reconstructed potential from measurements. The fundamental reason for this behavior is that the non-compact Laplace operator the "amplifies" the high-frequency error components if the forward equation is used to reconstruct the source, $Q(x)$, from measurements of the potential, $V(x)$.

The procedures used to solve approximately an illposed problem such as that described above are called regularization procedures (methods), after the systematic works by Philipps (Phillips 1962) and, who obtained "optimal solutions" by solving the minimization problem for a "cost functional", $F(x)$, containing user-defined parameters and meant to minimize a user-defined "error"; usually, this minimization problem takes on the form

$$
\operatorname{Min}_{x}[F(x)], \quad F(x) \equiv|A x-d|^{2}+\beta\left\|x-x_{0}\right\|^{2}
$$

where the Lagrange-like multiplier $\beta$ is a "free parameter" meant to accomplish a "user-defined compromise" between two requirements: (i) to satisfy the model equation $A x-d=0$, and (ii) to be close to the a priori knowledge $x_{0}$. A rich literature (too numerous to cite here) of variations on the Tichonov-Philips regularization procedure has since emerged; their common characteristic is the fundamental dependence of the "regularized solution" on "user-tunable" parameters, like $\beta$ in Eq. (25).

In an attempt to eliminate the appearance of "user-tunable parameters", Cacuci and co-workers (Barhen et al. 1980) combined concepts from information theory and Bayes' theorem to calibrate ("adjust") simultaneously system (model) responses and parameters, in order to obtain best-estimate values for both the responses and system parameter, with reduced uncertainties, with applications to reactor physics and design. Several years later, these methods (Barhen et al. 1980; Barhen et al. 1982) were rediscovered by workers in other fields, e.g., earth and atmospheric sciences (Barhen et al. 1982), mechanics of materials (Bui 1994), environmental sciences (Faragó et al. 2014), etc. We are given a functional result and asked to design the 
system to make that happen. For large-scale complex systems, it is practically impossible to run all possible cases in the "forward" direction (even with multivariate optimization algorithms) or to solve the inverse problem. Adjoint methods, which stem from Lagrange's method of "integration by parts" ( 1755), and were set on a rigorous mathematical foundation by Hilbert and Banach, were used (already in the 1940s) for solving efficiently linear problems in nuclear and reactor physics, and (a decade later) optimal control, by avoiding the need to solve repeatedly forward or inverse problems with altered model parameter values. However, these early adjoint methods were applicable solely to linear problems, since nonlinear operators do not admit "adjoints", as is universally known. Cacuci and co-workers (Cacuci et al. 1980a; Cacuci et al. 1980b) initiated the application of adjoint methods for computing sensitivities of simple responses in simple nonlinear problems. In a remarkable breakthrough, Cacuci (Cacuci 1981a; Cacuci 1981b; Cacuci 1988) developed in 1981 a mathematically-rigorous "adjoint sensitivity analysis" theory applicable to completely general nonlinear systems. Since the late 1980s, adjoint methods enjoyed a remarkably fast and wide-spread field of applications, from interpretation of seismic reflection data (Yedlin \& Pawlink 2011), to airfoil design (e.g., the Boeing 747 wing, (Kress et al. 1991)), to numerical error control (Kress et al. 1991).

In recent years, Cacuci and co-workers (Cacuci 2003; Cacuci et al. 2005; Cacuci and Mihaela Ionescu-Bujor 2010; Cacuci et al. 2014; Cacuci 2014a) have embarked on an effort to formulate a new conceptual framework that unifies the currently disparate fields of "inverse problems", data assimilation, model calibration and validation, by developing a unified framework based on physics-driven mathematical procedures founded on the maximum entropy principle, dispensing with the need for "minimizing user-defined cost functionals" (which characterizes virtually all of the methods currently in use). This fairly self-explanatory framework is depicted in Figure 5, and aims at developing validated predictive computational methods that can be used in the design of multi-phase HeteroFoaM materials. Such methods will be capable of designing not only the constituent materials and their interactions, but also the morphology of the shape, size, surfaces and interfaces that define the heterogeneity and the resulting functional response of the material system. Last but not least, this framework is envisaged to provide the foundation for developing game-changing high-order (to at least fourth-order, including skewness- and kurtosis-like moments of the predicted distributions for design parameters and responses of interest) predictive direct and inverse modelling capabilities, empowered by a new high-order adjoint sensitivity analysis procedure (HO-ASAP) for computing exactly and extremely efficiently ("smoking fast") response sensitivities of arbitrary order to any and all parameters in large-scale coupled multi-physics models. The high efficiency of the second-order adjoint sensitivity analysis procedure (SO-ASAP) has been illustrated (Cacuci 2014b) via an application to a paradigm particle diffusion problem; a series of papers documenting the HO-ASAP are currently in preparation.

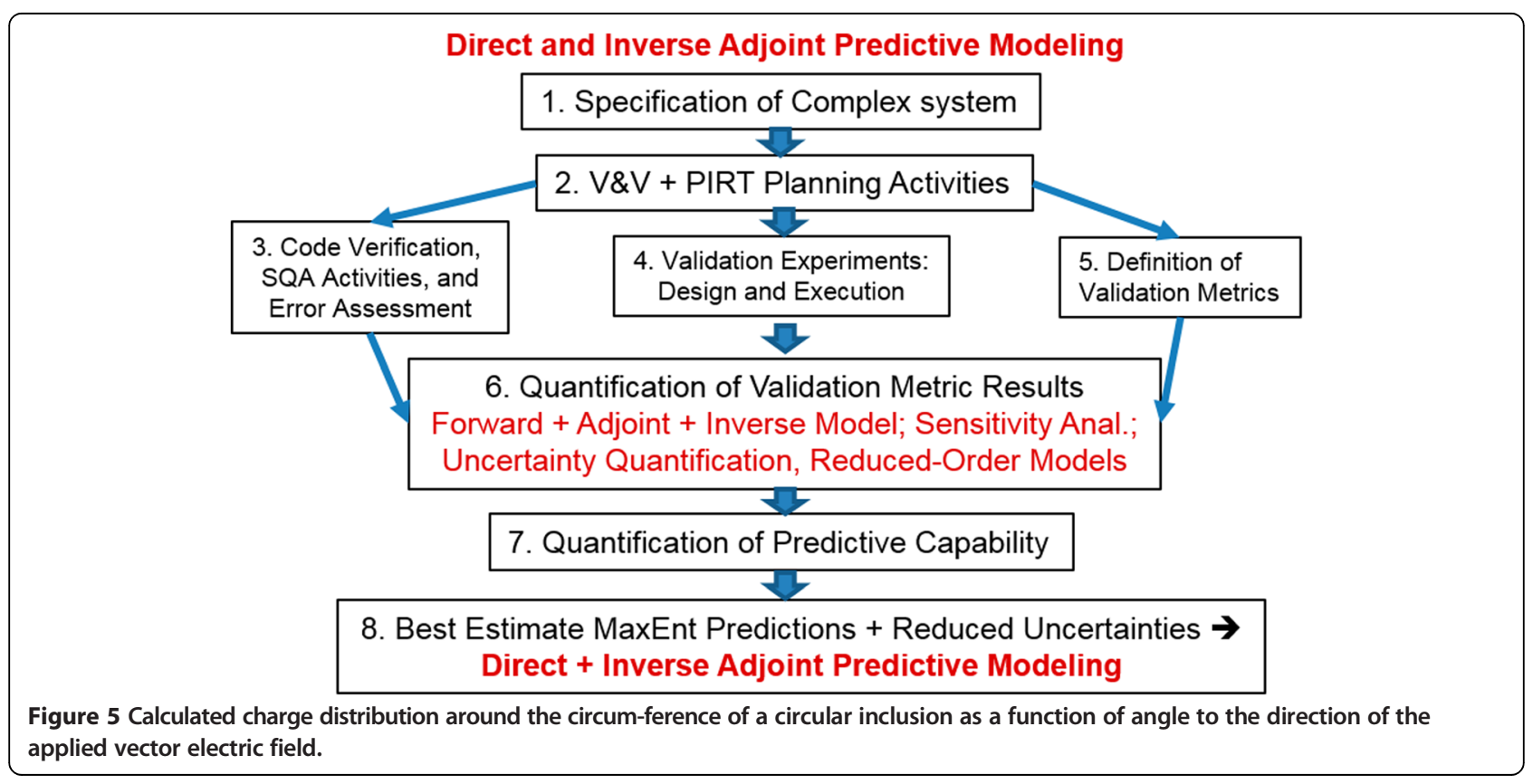




\section{Conclusions}

The present paper has outlined a foundation for developing validated predictive computational methods that can be used in the design of a genre of multi-phase heterogeneous functional materials that we call HeteroFoaM. We have defined and discussed analysis methods that will be capable of designing not only the constituent materials and their interactions, but also the morphology of the shape, size, surfaces and interfaces that define the heterogeneity and the resulting functional response of the functional material system. We have also discussed applications which drive this development. The problem of designing a heterogeneous functional material for specified dielectric performance, specifically addressing the role of space charge at heterogeneous interfaces, was presented as an example of the multi-scale functional behaviour that drives this approach and the methodology discussed.

\section{Competing interests}

The authors declare that they have no competing interests.

\section{Authors' contributions}

KR originated the heterogeneous functional materials ("HeteroFoaM ${ }^{\mathrm{TM}}$ ) concept and drafted the background and experimental sections of the manuscript. DC contributed to the predictive design concepts and wrote the analysis section on design for response. JB and JMA conducted the atomic force microscrope experiments and contributed to the interpretation of those data. FR conducted multiphysics analysis in support of interpretation of the data and constructed models of the experimental specimens in COMSOL. All authors read and approved the final manuscript.

\section{Authors' information}

Principal author Reifsnider is Director of the South Carolina SmartState Center for Solid Oxide Fuel Cells and a University Professor of Mechanical Engineering at the University of South Carolina.

\section{Acknowledgements}

The authors gratefully acknowledge the support of the broadband dielectric spectroscopy and related work by the Energy Frontier Research Center for Heterogeneous Functional Materials, the HeteroFoaM Center, under DoE Grant no.DE-SC0001061 from the Office of Basic Energy Sciences. Instrumentation and laboratory facilities used in the execution of the reported work are maintained by the South Carolina SmartState Center for Solid Oxide Fuel Cells and the Department of Mechanical Engineering at the University of South Carolina.

Received: 28 August 2014 Accepted: 25 November 2014 Published online: 05 May 2015

\section{References}

Baker J, Adkins JM, Rabbi F, Liu Q, Reifsnider K, Raihan R, (2014) Meso-Design of heterogeneous dielectric material systems: structure property relationships, Journal of Advanced Dielectrics, 4:1450008

Barhen J, Cacuci DG, Wagschal JJ, Mullins CB (1980) A systematic methodology for the reduction of uncertainties in transient thermal-hydraulics by using in-bundle measurement data". ANS Topical Conference 1980 Advances in Reactor Physics and Shielding, Sun Valley, Idaho, pp 156-168, September 14-17, 1980, ANS/70048

Barhen J, Cacuci DG, Wagschal JJ, Bjerke MA, Mullins CB (1982) Uncertainty analysis of time-dependent nonlinear systems: theory and application to transient thermal-hydraulics. Nucl Sci Eng 81:23-44

Bui HD (1994) Inverse problems in the mechanics of materials: an introduction. CRC Press Inc, Boca Raton, USA

Cacuci DG (1981a) Sensitivity theory for nonlinear systems: i. nonlinear functional analysis approach. J Math Phys 22:2794-2802
Cacuci DG (1981b) Sensitivity theory for nonlinear systems: ii. extensions to additional classes of responses. J Math Phys 22:2803-2812

Cacuci DG (1988) The forward and the adjoint methods of sensitivity analysis. Chapter 3 in Uncertainty Analysis, Ronen Y (ed). CRC Press, Inc, Boca Raton, Florida, pp 71-144

Cacuci DG (2003) Sensitivity and Uncertainty Analysis: Theory. 1. Chapman \& Hall/ CRC, Boca Raton

Cacuci DG (2014a) Predictive modeling of coupled multi-physics systems: I. theory. Annals of Nuclear Energy 70:266-278

Cacuci DG (2014b) Efficient and exact computation of second-order response sensitivities using adjoint systems: a paradigm illustrative neutron diffusion problem. Trans. Am. Nucl. Soc., Track \# 11528, Anaheim, CA, November 9-13

Cacuci DG, Weber CF, Oblow EM, Marable JH (1980a) Sensitivity theory for general systems of nonlinear equations. Nucl Sci Eng 75:88-110

Cacuci DG, Greenspan E, Marable JH, Williams ML (1980) Developments in sensitivity theory. ANS Topical Conference "1980 Advances in Reactor Physics and Shielding", Sun Valley, Idaho, September 14-17, 1980, NAS/ 70048: 692-704. See also: Cacuci DG, Weber CF (1980) Application of sensitivity theory for extrema of functions to a transient reactor thermal-hydraulics problems. Trans. Am. Nucl. Soc. 34: 312

Cacuci DG, lonescu-Bujor M, Navon Ml, (2005) Sensitivity and Uncertainty Analysis: Applications to Large Scale Systems. 2, Chapman \& Hall/CRC, Boca Raton, Florida, USA

Cacuci DG, Navon MI, lonescu-Bujor M (2014) Computational Methods for Data Evaluation and Assimilation. Chapman \& Hall/CRC, Boca Raton, Florida

D.G. Cacuci and Mihaela Ionescu-Bujor, "Sensitivity and Uncertainty Analysis, Data Assimilation and Predictive Best-Estimate Model Calibration", (2010), Chapter 17 in Vol.3, pp 1913 - 2051, Handbook of Nuclear Engineering, D. G. Cacuci, Editor, ISBN: 978-0-387-98150-5, Springer Verlag, New York, Berlin/Heidelberg 2010.

Faragó I, Havasi A, Zlatev Z (eds) (2014) Advanced numerical methods for complex environmental models: needs and availability. Publishers, Bentham Science

Kress R, Lassi P, Arinta A (1991) On the far field in obstacle scattering. SIAM, J. Appl. Math

Liu Q (2011a) Physalis method for heterogeneous mixtures of dielectrics and conductors: Accurately simulating one million particles using a PC. J Comp Phys 230:8256-8274

Liu Q (2011b) Physalis method for heterogeneous mixtures of dielectrics and conductors: accurately simulating one million particles using a PC. J Comp Phys 230:8256-8274

Liu Q (2012) Directly resolving particles in an electric field: local charge, force, torque, and applications. Int J Numer Meth Engng 90:537-568

Liu Q, Reifsnider K (2013) Heterogeneous mixtures of elliptical particles: directly resolving local and global properties and responses. J Comp Phys 235:161-181

Phillips DL (1962) A technique for the numerical solution of certain integral equations of the first kind. J Assoc Comp Mach 9:84-97

Raihan R, Adkins J-M, Baker J, Rabbi F, Reifsnider K (2014) Relationship of Dielectric Property Change to Composite Material State Degradation, Composites Science and Technology. http://dx.doi.org/10.1016/j. compscitech.2014.09

Reifsnider K, Chiu WK, Brinkman K, Du Y, Nakaja A, Rabbi F, Liu Q (2013) Multiphysics design and development of heterogeneous functional materials for renewable energy devices: the HeteroFoaM story. J. Electrochemical Society 160:F470-F481

Yedlin M, Pawlink P (2011) Scattering from cylinders using the two-dimensional vector plane wave spectrum. J Opt Soc Am A 28(6):1177-1184 doi:10.1364/josaa.28.001177 